\title{
Violencia intrafamiliar y de género en los beneficiarios del Proyecto de Circo Social - Cuenca
}

\section{Domestic and gender violence in the beneficiaries of the Social Circus Project - Cuenca}

\author{
Janeth Nataly Urgiles-Palomeque \\ janeth.urgiles@psg.ucacue.edu.ec \\ Universidad Católica de Cuenca, Azogues \\ Ecuador \\ https://orcid.org/0000-0002-3015-2123 \\ Darwin Gabriel García-Herrera \\ dggarciah@ucacue.edu.ec \\ Universidad Católica de Cuenca, Azogues \\ Ecuador \\ https://orcid.org/0000-0001-6813-8100 \\ Sergio Constantino Ochoa-Encalada \\ scochoae@ucacue.edu.ec \\ Universidad Católica de Cuenca, Cuenca \\ Ecuador \\ https://orcid.org/0000-0003-3067-3719 \\ Juan Carlos Erazo-Álvarez \\ jcerazo@ucacue.edu.ec \\ Universidad Católica de Cuenca, Cuenca \\ Ecuador \\ https://orcid.org/0000-0001-6480-2270
}

Recepción: 08 abril 2020

Revisado: 16 de mayo 2020

Aprobación: 16 junio 2020

Publicación: 1 de julio 2020 


\title{
RESUMEN
}

El objetivo de este estudio es conocer las formas en las que se da la violencia intrafamiliar asociada al género. Se utilizó en la metodología un enfoque cuantitativo, de tipo descriptivo no experimental y de cohorte transversal. Los resultados corroboraron la existencia de violencia en distintas formas, predominando la violencia sexual. El estudio ha develado que las usuarias del Proyecto Circo Social no identifican ciertas acciones como violencia, pues al responder si su pareja es violenta su respuesta es negativa. Sin embargo, son conscientes de que algunas veces realizan acciones que están sujetas a la aprobación o desaprobación de su pareja. Además. Se menciona que la mayor parte de las participantes su cónyuge consume sustancias psicotrópicas, lo cual se enlaza con la generación de violencia dentro del círculo familiar asociada al género, considerándose como una base de la desestructuración familiar y matrimonial.

Descriptores: Violencia doméstica; relación padres-hijos; abuso sexual; discriminación. (Palabras tomadas del Tesauro UNESCO).

\begin{abstract}
The objective of this study is to know the ways in which gender-related intra-family violence occurs. A quantitative, descriptive, non-experimental, cross-sectional approach was used in the methodology. The results corroborated the existence of violence in different forms, with sexual violence prevailing. The study has revealed that users of the Social Circus Project do not identify certain actions as violence, since when they answer if their partner is violent, their response is negative. However, they are aware that sometimes they perform actions that are subject to the approval or disapproval of their partner. Also. It is mentioned that most of the participants their spouse consumes psychotropic substances, which is linked to the generation of violence within the family circle associated with gender, being considered as a basis for family and marital breakdown.
\end{abstract}

Descriptors: Domestic violence; parent child relationship; sexual abuse; discrimination. (Words taken from the UNESCO Thesaurus). 


\section{INTRODUCCIÓN}

La familia se ha consolidado en el medio como la unidad básica de la sociedad, por lo que se constituye como el núcleo fundamental para el desarrollo del individuo. En este sentido, en el Ecuador según el Instituto Nacional de Estadísticas y Censos [INEC], destaca la inexistencia de documentación e investigación sobre la violencia doméstica, hostigamiento sexual y la violencia contra mujeres y niñas, en los ámbitos públicos, privados. Por lo tanto, no existe cifras o índices estadísticos establecidos que ayuden a visibilizar el fenómeno; no obstante, la defensoría pública presta servicios gratuitos para el asesoramiento, intervención y ha establecido rutas de protección en casos de violencia intrafamiliar. Entonces, surge una paradoja sobre el fenómeno, en la cual se basa esta investigación y consolida su problema a investigar.

En esta investigación primero definimos la exposición a la violencia y luego presentamos datos de internacionales y nacionales sobre la prevalencia de la exposición a la violencia, que muestran que estar expuesto a la violencia es una experiencia común para muchos niños en países industrializados, y se discuten las principales consecuencias asociadas con la exposición a violencia, en todas sus formas y en todos los contextos. A continuación, nos centraremos en el tema específico de la violencia intrafamiliar en el contexto cuencano, incluyendo algunos mecanismos a través de los cuales la violencia podría afectar el desarrollo infantil y los factores de riesgo y protección que se sabe que aumentan o reducen la probabilidad de un desarrollo infantil (Conger, 2003).

En el Ecuador se considera a la violencia intrafamiliar vinculada al género se ha considerado como una de las problemáticas sociales más contundentes de las últimas décadas. Por lo que se han dado álgidos debates que han girado en torno a los derechos humanos, los cuales exigen al estado la rectoría de este tema. Según la Constitución de la República del Ecuador (2008) Art. 35 “... garantiza atención prioritaria a las personas en situación de riesgo, las víctimas de violencia doméstica y sexual...". Del mismo modo, en el (Codigo Organico Integral Penal [COIP ], 2014)Art. 157 la 
persona que ejerza violencia contra la mujer o miembros del núcleo familiar, se sancionará dependiendo la magnitud de la agresión, desde pena preventiva hasta tres años de prisión.

Con base al régimen normativo que garantiza la protección de los derechos y sanciona las acciones vinculadas a la violencia doméstica, se justifica la necesidad de abordar esta problemática, con una visión en el mejoramiento de la calidad de vida de las mujeres víctimas de dicho fenómeno, por medio de la ejecución de proyectos psicoeducativos y psicosociales basados en el enfoque de acción participativa. La relevancia del estudio yace en la existencia de cifras que den cuenta de índices altos de violencia intrafamiliar vinculadas al género como una problemática social que incluso podría terminar con la muerte de la víctima.

Al referirnos a la pertenencia del estudio, es necesario remitirnos a los acontecimientos de conocimiento público sobre los diferentes casos de violencia registrados por la Fiscalía General del Estado 2019 y 12 mil casos de violencia escolar registrado por el (Ministerio de Educacion del Ecuador, 2019), permiten contemplar la necesidad de conocer las diferentes aristas y vínculos que surgen de estas dos variables de análisis. Para ello, se demostró estadísticamente la fiabilidad de los datos de acuerdo al Alfa de Cronbach de 0,9 el cual equivale a una valoración alta de acuerdo a los estándares estadísticos.

La violencia intrafamiliar asociada al género se ha normalizado culturalmente y las transformaciones sociales actuales, han generado el desarrollo de una serie acontecimientos disruptivos que afecta el desarrollo personal e integral del individuo. Las distintas realidades observadas en el Proyecto Circo Social al tema de la violencia intrafamiliar vinculada al género y la experiencia directa o indirecta que han tenido las usuarias han motivado la realización de la investigación, con miras a la generación de proyectos psicoeducativos y psicosociales en beneficio de esta población en situación de vulneración. Con relación a la realidad del Proyecto Circo Social del Cantón Cuenca, relacionado a los procesos de intervención psicoeducativa y psicosocial, dan cuenta de 
la necesidad de abordar la violencia intrafamiliar como un detonante de la desestructuración familiar de los usuarios, los mismos que se componen de diferentes características, tales como: pobreza extrema, hacinamientos, entre otros.

En la investigación sobre la violencia intrafamiliar o doméstica relacionada a estrategias de afrontamiento, se distinguen autores como: (Hernández, 2018) que mencionan que es un problema social, en la cual niños y jóvenes están expuestos directa 0 indirectamente, por lo que se hace mención de que es necesario un apoyo amplio de toda la sociedad hacia los más vulnerables. En la actualidad, con el transcurso del modernismo y la globalización se ha perdido la esencia básica de la familia; no obstante, aún permanecen los constructos sociales a nivel de Latinoamérica, que restringe los derechos de las mujeres, niños y niñas; por lo que se ha llegado a normalizar la violencia a tal punto de no ser las personas capaces de empoderarse de sí mismas (Espinar-Ruiz, 2003).

La violencia es generalizada en el mundo, incluso entre quienes viven en los países más desarrollados económicamente. En muchas ciudades industrializadas occidentales, a uno de cada dos niños cada año se le niega su derecho fundamental a ser protegido de la violencia y crecer en hogares, escuelas y comunidades saludables y seguras, con una exposición temprana a la violencia que probablemente sea especialmente en detrimento del bienestar del niño (McClennen \& Day, 2016).

La violencia que invade las sociedades occidentales afecta el desarrollo integral y el funcionamiento psicológico de muchas maneras similares. Un postulado básico es que la violencia engendra violencia, pero muchos de los casos no se llegan a identificar por la víctima. Además, las personas expuestas a la violencia tienen un mayor riesgo de ansiedad y depresión, principalmente debido a la percepción de peligro en el contexto donde viven. Por último, una exposición repentina o crónica a la violencia puede provocar síntomas de trastorno de estrés postraumático (TEPT) (McClennen \& Day, 2016) como la re experimentación intrusiva e involuntaria de un evento traumático, alto nivel emocional despertar y evitar situaciones que recuerden el evento. 
Algunos autores como realizan una reflexión en torno a la desintegración familiar y los lazos afectos que se rompen cuando se suscitan dichas circunstancias. De tal manera se evidencia que las causas de la desorganización familiar prevalecen las adicciones por uno de los padres, por la pobreza extrema o la violencia familiar; concomitante a esto existe una alta coincidencia entre las causas que originan la agresividad y las manifestaciones conductuales, por lo que se llega a observar comportamientos impulsivos, baja autoestima, actitud desafiante y agresividad.

Cuando se menciona a la familia y específicamente la pareja, autores (López \& Guiamaro, 2017) centran su análisis en las estructuras familiares, los roles y las jerarquías; por lo tanto, manifiestan que los roles de los adultos deben estar bien definidos para que se ejecute un buen desarrollo de las habilidades comunicativas de la pareja; es por ello que se hace hincapié en las relaciones como símbolo de complicidad y empatía para la generación de habilidades como el asertividad sexual.

Hay que tomar en cuenta que la violencia intrafamiliar asociada a las diferencias de género no solo se da entre la pareja, sino que podría derivar hacia hijos del género opuesto. Entonces, se pudiera afirmar el comportamiento violento es multicausal, y que la finalidad última es aniquilar la vida de una o varias personas o poner un grave peligro su existencia. Con este postulado, el maltrato infantil directo o indirecto se concibe como un problema de salud pública, debido a los daños que ocasiona, dentro de los actos calificados como maltrato están los siguientes: el castigo físico o verbal, la humillación, el abuso físico o psicológico, el descuido y negligencia. En este sentido señalan que los actos que por acción u omisión desconocen los derechos fundamentales de los niños e interfieren su desarrollo integral y llegan o pueden llegar a poner en peligro su salud física, psicológica, social y sexual.

El fenómeno de la violencia ha sido estudiado desde diferentes perspectivas y áreas de la ciencia, sin embargo, la violencia intrafamiliar responde al análisis de las ciencias sociales; esta es definida como un tipo de abuso o agresión que yace dentro del círculo familiar y que se ejecuta entre la pareja que conforman el núcleo matrimonial, estas 
acciones pueden derivarse del mal funcionamiento de las relaciones, ya sea en su jerarquía que obedece a un patrón de los sistemas de relaciones de poder (Navarrete, 2011). Es por ello que, dentro del enfoque de derechos se da una visión holística que tiene como principio fundamental la igualdad.

La violencia ha sido responsable de más del 2,5\% de la población a nivel mundial. Estas cifras forman parte de la pirámide de mortalidad en las que se incluyen enfermedades, accidentes, entre otros. Es por ello que en el estudio realizado por (Ahlfs-Dunn, 2016) se discuten aspectos legislativos que sancionan la violencia intrafamiliar y el respetivo seguimiento que se realiza luego de una denuncia. Uno de los problemas más habituales para recurrencia del fenómeno es la falta de seguimiento y la forma en la que las víctimas desisten debido a la revictimización durante la recopilación de pruebas. Dado estos argumentos, el estudio concluye que se debería construir nuevas formas de inspección y de investigación, el cual conste una información complementaria y totalitaria.

A raíz de los cambios y transformaciones que ha sufrido la familia actual, a nivel estructural, funcional y vital, con respecto a la diversidad y la complejidad del núcleo marital, es imposible generar una definición única del concepto pareja. Con las transformaciones sociales, las políticas públicas plantean una preocupación latente en las interacciones sociales y sus desafíos, debido a los cambios experimentados en el ámbito social. (Casavilca, 2019), manifiesta la evolución de la pareja viene dada por las transformaciones y constructos sociales que determinan el deber ser del ideal de pareja, sin embargo, con la dominancia del sistema machista se ha dejado huellas imborrables para que se dé un buen estado marital en armonía. Para ello es fundamental realizar cambios de base manifiesta.

Con respecto a las relaciones jerárquicas, se establece un modelo patriarcal en el que se da la violencia como resultado de la búsqueda de poder de la una persona sobre la otra. Las asimetrías en las relaciones determinan la forma en cómo se vive la convivencia, por lo tanto, es necesario considerar un enfoque relaciones de poder que 
funcionan bajo tres dimensiones: la dimensión simbólica que se refiere a las representaciones múltiples, o sea mitos sociales construidos; la dimensión normativa que representa a las interpretaciones de los significados de los símbolos y que se expresan en doctrinas religiosas, educativas, legales y políticas, que su vez ubican el ser varón o mujer en cada contexto; y finalmente, la dimensión sistémica se refiere a las instituciones y organizaciones sociales que es el sistema de parentesco, el educativo, económico y político (Guillén, 2004)

La investigación de (Amar \& Berdugo de Gómez, 2011) evidencia que a mayor precisión y consistencia los factores psicosociales mediante los cuales los niños víctimas de violencia intrafamiliar activa y/o pasiva pueden desarrollar una personalidad resiliente, debido a mecanismos psicológicos que lo fortalecen de una forma plástica. Es decir que, a mayores dificultades experimentadas por los niños, mayor es el nivel de resiliencia que pueden generar, y al mismo tiempo, una reestructuración cognitiva que fortalece su visión del mundo.

Sin embargo, la violencia no solo está centrada en una estructuración interna familiar, sino que comprende también de los factores psicosociales, tales como: adicciones de padres y/o hijos, la extrema pobreza o un círculo de violencia transgeneracional. Del mismo modo factores biológicos en las personas violentas, mismas que tienden a tener conductas distorsionadas en los mensajes y percibirlos como una provocación, por lo que los lleva actuar de una manera impulsiva o que ocasiona conductas más comunes en niños de entre 5 a 7 años, debido a que se produce una descarga hormonal que produce un incremento de los andrógenos que se asocian con la agresividad en niños. 


\section{METODO}

La investigación se fundamenta en la corriente de pensamiento del positivismo, por lo que se adoptará la modalidad cuantitativa y de alcance descriptivo no experimental, con la finalidad de medir e identificar las características del fenómeno a estudiar, cuantificar los resultados representativos para la población de estudio, siendo corte transversal, por cuanto se ubica en un espacio y tiempo específico (Erazo \& Narváez, 2020).

El método a utilizar es hipotético-inductivo, se partió de un supuesto teórico de lo general a lo específico. Dentro de los instrumentos a utilizar, primero se firmaron los consentimientos, se aplicó la escala de medición de la violencia intrafamiliar VIFJ4 modificada, la cual está compuesta por 12 preguntas y evalúa cinco áreas de la violencia intrafamiliar asociada al género, siendo estandarizado y no necesitando ser calculada su confiabilidad. La encuesta aplicada fue presencial en el domicilio de cada uno de las madres de los beneficiarios que forman parte del Proyecto Circo Social del Cantón Cuenca. (Calle-Abril, Erazo-Álvarez, \& Narváez-Zurita, 2020). El instrumento cuenta con cinco factores: violencia física, violencia psicológica, violencia sexual, violencia social y violencia patrimonial. En relación a las técnicas que se aplicaron, las escalas de autoinforme, el investigador debe incentivar a las personas para que complete su aportación con la investigación.

Para la muestra poblacional se consideró 20 madres que pertenecen al Proyecto Circo Social y tener una presunción de violencia intrafamiliar vinculada a género, aplicándose aspectos éticos que garantizaron la confidencialidad de los participantes precautelando la utilización de los datos, aspecto obedece al principio de no maleficencia. La participación fue voluntaria y de forma anónima, sin embargo, se requerió la firma del consentimiento informado y asentimientos informados (Mendieta-Ortega, Erazo-Álvarez, \& Narváez-Zurita, 2020). Para el análisis de los datos, se empleó el Software SPSS, en el cual se realizaron las tablas y gráficos que ayudaron a la estructuración de los resultados. 


\section{RESULTADOS}

En este apartado de la investigación se describen los aspectos más relevantes en cuanto a los resultados de la investigación según las variables operacionales del instrumento utilizado. En este mismo sentido, evidencian los siguientes resultados:

Con respecto al instrumento se dividía en seis factores; el primero relacionado con la violencia física para lo cual se aplicaron tres preguntas según la siguiente escala de Likert: casi nunca, pocas veces, a veces, muchas veces y casi siempre. De este modo se evidencia que el $40 \%$ de las participantes califican como 'Casi nunca' su pareja ha sido agresivo; en los resultados se muestran puntuaciones bajas en agresividad. Bajo la misma perspectiva, el $60 \%$ de las participantes manifiesta que no ha sangrado a causa de golpes. Finalmente, el $70 \%$ no ha tenido que recurrir a atención médica a causa de golpes recibidos. De esta forma, se obtienen una desviación estándar que oscila desde 0,82 hasta 0,92 , lo cual muestra una sincronía en los datos reflejados.

\section{Tabla 1}

Violencia física

\begin{tabular}{|c|c|c|c|}
\hline & $\begin{array}{l}\text { ¿Su pareja es } \\
\text { agresiva? }\end{array}$ & $\begin{array}{c}\text { ¿Ha sangrado a causa } \\
\text { de golpes recibidos por } \\
\text { su pareja? }\end{array}$ & $\begin{array}{l}\text { ¿Por los golpes ha } \\
\text { recibido atención } \\
\text { médica? }\end{array}$ \\
\hline Nro. Válidos & 20 & 20 & 20 \\
\hline Perdidos & 0 & 0 & \\
\hline Media & 1,95 & 1,55 & 1,45 \\
\hline Mediana & 2,00 & 1,00 & 1,00 \\
\hline Desviación estándar & 0,045 & 0,826 & 0,826 \\
\hline Asimetría & 0,524 & 1,695 & 2,047 \\
\hline $\begin{array}{l}\text { Error estándar de } \\
\text { asimetría }\end{array}$ & 0,512 & 0,512 & 0,512 \\
\hline
\end{tabular}

Fuente: Elaboración propia.

En segunda instancia se devela la variable operacional de violencia psicológica en la cual los participantes manifestaron lo siguiente: al $45 \%$ de las participantes su pareja valora su opinión; el $50 \%$ no ha recibido amenazas de suicidio en caso de abandono; y el $45 \%$ casi no tiene temor de su pareja, cuando llega a la casa. Esto significa que los datos develan una curva de distribución normal, la misma que sitúa la misma cantidad 
de datos a su derecha y a su izquierda, ya que se evidencia una desviación estándar que oscila entre 1,071 y 0,605 .

\section{Tabla 2}

Violencia psicológica

\begin{tabular}{lrrr} 
& $\begin{array}{c}\text { ¿Su pareja le suele } \\
\text { hacer callar cuando } \\
\text { usted da su opinión? }\end{array}$ & $\begin{array}{c}\text { ¿Su pareja ha } \\
\text { amenazado con } \\
\text { suicidarse si le } \\
\text { abandona? }\end{array}$ & $\begin{array}{c}\text { ¿Usted tiene temor } \\
\text { cuando su pareja llega } \\
\text { a la casa? }\end{array}$ \\
\hline Nro. Válidos & 20 & 20 & 20 \\
Perdidos & 0 & 0 & 0 \\
Media & 1,90 & 1,55 & 1,85 \\
Mediana & 2,00 & 1,50 & 1,00 \\
Desviación estándar & 1,071 & 0,605 & 0,988 \\
Asimetría & 1,073 & 0,583 & 1,056 \\
Error estándar de & 0,512 & 0,512 & 0,512 \\
asimetría & & & \\
\hline
\end{tabular}

Fuente: Elaboración propia.

Para el factor de violencia sexual se ha tomado en consideración dos preguntas que hacen referencia al no respeto del consentimiento mutuo para las relaciones sexuales y a la permisibilidad del uso de anticonceptivos. Con respecto al primer aspecto el $15 \%$ de las participantes responden que a veces su pareja le obliga a tener relaciones sexuales cuando ella no desea; y el $5 \%$ se siente muchas veces obligada. Con la segunda pregunta, existe un $10 \%$ que responde a veces; un $10 \%$ muchas veces y un $5 \%$ casi siempre su pareja le prohíbe el uso de anticonceptivos. Además, se muestra una dispersión de datos que oscila entre 0,93 y 1,191. 


\section{Tabla 3}

Violencia sexual

¿Su pareja le obliga a tener relaciones sexuales cuando usted no desea?

Nro. Válido

Perdidos

Media

Mediana

Desviación estándar

Asimetría

Error estándar de asimetría
¿Su pareja le prohíbe el uso de métodos anticonceptos?

\section{Fuente: Elaboración propia}

En el análisis de la violencia social se contemplaron dos preguntas relacionadas al impedimento por parte de la pareja en cuanto al cuidado físico y las relaciones sociales mediante el uso de dispositivos móviles. En el primer apartado se evidencia que el $25 \%$ de las participantes a veces siente que su pareja le impide el cuidado de su arreglo físico; mientras que el $20 \%$ y $15 \%$ manifiesta que muchas veces o casi siempre su pareja le impide hablar con otras personas por medio del celular. Concomitante a esto, se encuentra una dispersión de los datos de 1,070 y 1,56 respectivamente, lo cual muestra una alta concordancia entre sí.

\section{Tabla 4}

Violencia social

¿Su pareja se pone molesto cuando se arregla?
¿Su pareja le impide hablar con otras personas por celular?

\begin{tabular}{lcc}
\hline Nro. Válido & 20 & 20 \\
Perdidos & 0 & 0 \\
Media & 2,25 & 2,20 \\
Mediana & 2,00 & 2,00 \\
Desviación estándar & 1,070 & 1,058 \\
Asimetría & 0,877 & 0,453 \\
Error estándar de asimetría & 0,512 & 0,512 \\
\hline
\end{tabular}

Fuente: Elaboración propia 
En cuanto a la violencia patrimonial se muestra que el $25 \%$ de los agresores ha roto 0 destruido las cosas dentro del hogar y $15 \%$ ha impedido el ingreso de la víctima a su domicilio. Los datos presentan una curva de dispersión normal, lo cual evidencia una asimetría con tendencia media-superior.

\section{Tabla 5}

Violencia patrimonial

\begin{tabular}{lcc} 
Nro. Válido & $\begin{array}{c}\text { ¿Su pareja ha roto las cosas del } \\
\text { hogar? }\end{array}$ & $\begin{array}{c}\text { ¿Su pareja le ha impedido el } \\
\text { ingreso a su domicilio? }\end{array}$ \\
\hline Perdidos & 20 & 20 \\
Media & 0 & 0 \\
Mediana & 2,00 & 2,05 \\
Desviación estándar & 2,00 & 2,00 \\
Asimetría & 0,076 & 1,040 \\
Error estándar de asimetría & 0,563 & 1,576 \\
\hline
\end{tabular}

Fuente: Elaboración propia

Como parte de la investigación se tomó con un aspecto importante al hecho de consumo de sustancias psicotrópicas en la ocurrencia de la violencia intrafamiliar ligada al género. Para ello se preguntó si el agresor consumía o no consumía sustancias. Ante este aspecto se encontró que el 55\% de las dos variables operacionales relacionadas al consumo de alcohol. Con este antecedente, se realizó un cruce de variables entre la violencia sexual y el consumo.

De acuerdo a los datos presentados, se evidencia una relación directa entre el consumo de sustancia y la violencia sexual dando como resultado una concordancia del $55 \%$ de coincidencia. En este sentido, se halló una verosimilitud de 3,54 y se encontró una verosimilitud de 6, 19 con una discrepancia de los resultados mayor o igual a 4,99 en la variable de impedimento en el uso de anticonceptivos.

Con respecto a las demás variables operacionales se encuentra un nivel de significancia que oscila entre 0,01 y 0,03; que evidencia unas significancias asintóticas menos de 0,05 de acuerdo a la prueba no paramétrica. 
A partir de los resultados anteriormente mencionados, es necesario realizar una discusión con breve contrarrestando la evidencia empírica más la teoría. Para ello, es importante abordar la violencia intrafamiliar asociada al género desde la perspectiva de derechos; en este sentido se menciona los derechos constitucionales que garantizan la protección a las víctimas de violencia en cualquiera de sus formas (Rico, 1996).

De acuerdo a los que las participantes mencionan, muy pocas de ellas identifican ser víctima de violencia; sin embargo, cuando se les pregunta sobre aspectos relacionados a los cinco factores de la escala aplicada no existe una respuesta concordante a la percepción de no ser víctima de violencia. Según (Casavilca, 2019) cuando la violencia no llega a ser tan agresiva, la víctima suele no reconocerla, pero cabe mencionar que por más sutil que sea no deja de ser una vulneración de derechos.

Uno de los aspectos más relevantes de la investigación está relacionado con el ámbito sexual de la pareja. Culturalmente en la sociedad ha sido mal visto que una mujer hable se sexualidad abiertamente; por ello aún en la actualidad muchas mujeres se sienten forzadas a tener intimidad cuando no lo desean, no siempre el hombre obliga de forma directa, sino que la mujer no es capaz de expresarse frente a la situación (Ames \& Crisóstomo, 2019). Pese a que número poblacional investigado es pequeño, se ha mostrado que más del $50 \%$ de aquellas mujeres se sienten forzadas en el acto sexual.

A más de lo explicado, existe una relación directa entre la violencia y el consumo de sustancias. Por ello, (McClennen \& Day, 2016) hace mención que muchas desestructuraciones familiares no son causa de la violencia como tal, sino que existen factores base que incitan dicha violencia; como es el consumo de sustancias de un miembro del hogar. Por lo tanto, desde este punto de vista se concuerda que la violencia es solo un efecto de factores sociales, personales, entre otras; que son propios del agresor. 


\section{PROPUESTA}

Se propone la realización de un micro proyecto "Unidos con amor" basado en la metodología de acción participativa IAP, dónde los participantes sean actores principales de la propia construcción de sus conocimientos basados en las problemáticas propias de cada pareja. Del mismo modo, es relevante y pertinente el abordaje de dicho fenómeno, ya que para el macro Proyecto Circo Social es fundamental la convivencia armónica de las familias que son beneficiarias de procesos psicosociales y psicoeducativos. Es por ello, que se realiza está propuesta de afianzar lazos afectivos y/o emocionales que son clave para el fortalecimiento de las estructuras familiares; y de forma específica, de la pareja.

La finalidad de este el diseño y conducción de diferentes estrategias y acciones que desde la parte psicológica se desarrollan para prevenir, atender y limitar el daño asociado a casos de violencia familiar, sexual y contra las mujeres; por medio de la sensibilización y concientización.

\section{Tabla 6}

Micropoyecto Unidos con amor

Objetivo del proyecto:

Metodología:

Duración:

Coordinadora del proyecto:

Finalidad:
Generar lazos fuertes de afecto en las parejas que son beneficiarios del macro Proyecto Circo Social; y por ende, afianzar la unión de pareja y familiar basándose en el respeto mutuo y el conocimiento del otro.

Investigación acción participativa IAP

6 meses

Janeth Nataly Urgiles Palomeque

El micro proyecto busca que los usuarios afiancen sus lazos afectivos y emocionales para la formación de la unión de pareja y familiar sea óptima.

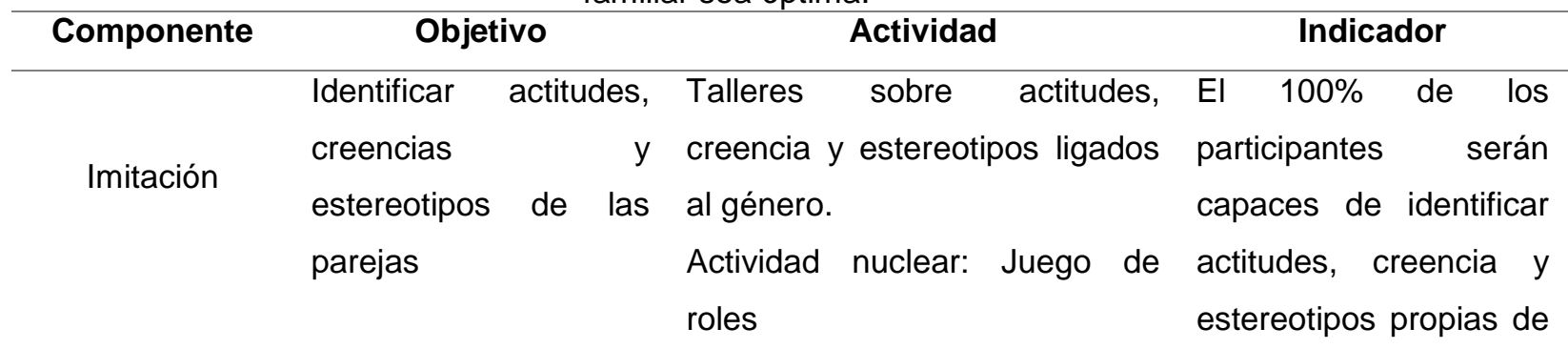


Janeth Nataly Urgiles-Palomeque; Darwin Gabriel García-Herrera; Sergio Constantino Ochoa-Encalada; Juan Carlos Erazo-Álvarez

\begin{tabular}{|c|c|c|c|}
\hline & & & su pareja. \\
\hline Afianzamiento & $\begin{array}{l}\text { Recordar los porqués } \\
\text { estoy junto con mi } \\
\text { pareja. }\end{array}$ & $\begin{array}{l}\text { Talleres de búsqueda de } \\
\text { motivos de la unión. } \\
\text { Actividad nuclear: línea de } \\
\text { tiempo. }\end{array}$ & $\begin{array}{l}\text { El } 100 \% \text { de los } \\
\text { participantes serán } \\
\text { capaces de encontrar } \\
\text { los motivos por los } \\
\text { cuales se realizó la } \\
\text { unión. }\end{array}$ \\
\hline Confrontamiento & $\begin{array}{l}\text { Evaluar los patrones } \\
\text { de riesgo de la } \\
\text { relación hablada, en la } \\
\text { que se incluye los } \\
\text { hijos. }\end{array}$ & $\begin{array}{l}\text { Talleres sobre los patrones de } \\
\text { riesgo y la resolución de } \\
\text { conflictos. } \\
\text { Actividad nuclear: FODA }\end{array}$ & $\begin{array}{l}\text { El } 100 \% \text { de los } \\
\text { participantes logrará } \\
\text { aprender estrategias } \\
\text { para la resolución de } \\
\text { conflictos. }\end{array}$ \\
\hline Conciliación & $\begin{array}{l}\text { Generar lazos } \\
\text { afectivos basados en } \\
\text { el aquí y ahora. }\end{array}$ & $\begin{array}{l}\text { Talleres sobre la atención plena } \\
\text { vinculada a la pareja. } \\
\text { Actividad nuclear: El vientre de } \\
\text { la madre. }\end{array}$ & $\begin{array}{l}\text { El } 100 \% \text { de los } \\
\text { participantes al terminar } \\
\text { los talleres serán } \\
\text { capaces de ser } \\
\text { conscientes de sus } \\
\text { propias acciones y de } \\
\text { los demás. }\end{array}$ \\
\hline
\end{tabular}

Fuente: Elaboración propia.

Para todas las actividades propuestas se requiere la coordinación holística de diferentes instituciones que proporcionen un carácter complementario e interdisciplinar, pues esto obliga a un planteamiento de atención que ha de incluir diferentes ámbitos de intervención y un trabajo sistemático con aquellos recursos y servicios presentes en la red externa Además, nos va a permitir dar una respuesta más integral ante la demanda específica de la familia, así como trabajar en otras facetas más preventivas en un futuro. 


\section{CONCLUSIONES}

El estudio ha develado que las usuarias del Proyecto Circo Social no identifican ciertas acciones como violencia, pues al responder si su pareja es violenta su respuesta es negativa. Sin embargo, son conscientes de que algunas veces realizan acciones que están sujetas a la aprobación o desaprobación de su pareja. Además. Se menciona que la mayor parte de las participantes su cónyuge consume sustancias psicotrópicas, lo cual se enlaza con la generación de violencia dentro del círculo familiar asociada al género, considerándose como una base de la desestructuración familiar y matrimonial. Por otra parte, la violencia de género se considera una de las implicaciones más fuertes, pues los detonantes de actos violentos se derivan de una asimetría en la jerarquía de roles y de poder.

Por otra parte, se hace hincapié en la violencia de género cuando específicamente se habla de sexualidad; este aspecto es relevante debido a que la mayor parte de participantes siente alguna vez esta aversión con sus parejas. Como se había comentado, uno de los patrones sobre el no respeto los derechos sexuales y reproductivos son dados por causas históricas-culturales; mismas que no solo responde a la responsabilidad masculina, sino que se considera un aspecto de corresponsabilidad entre la pareja.

Finalmente, como parte de la investigación se evidencia el siguiente hallazgo. Si bien es cierto que la violencia intrafamiliar y la violencia de género son dos aspectos relacionados, pero también son diferentes; sin embargo, cuando se habla de la pareja como una unidad estos tipos de violencia de alguna forma también se vuelve una unidad, que, a su vez, es igual o más compleja. Por ello, cuando se investiga la violencia intrafamiliar, solo tomando en cuenta a la pareja la problemática derivar a diferentes aristas bases de la desestructuración familiar, tomándola a esta como un sistema complejo de dinámicas cambiantes. 
Se recomienda para futuros estudios que estén guiados bajo la misma perspectiva, tomas en cuenta variables sociodemográficas como: estatus social, trabajo, edad, entre otros; que ayuden a vislumbrar este fenómeno ampliamente.

\section{FINANCIAMIENTO}

No monetario

\section{AGRADECIMIENTOS}

Al Proyecto Circo Social - Cuenca, por el apoyo en el desarrollo de la investigación.

\section{REFERENCIAS}

Ahlfs-Dunn, S. M.-B. (2016). Intimate partner violence involving children and the parenting role: Associations with maternaoutcomes [Violencia de pareja íntima que involucra a niños y el papel de los padres: asociaciones con resultados maternos]. Journal of family violence, 31(3), 387-399. doi:https://doi.org/10.1007/s10896-015-9791-x

Aldana-Zavala, J., \& Valles-González, E. (2018). La mujer en Derecho a una Vida libre de violencia desde la Organización Comunitaria. [Women in the Right to a Life free of violence from the Community Organization]. IUSTITIA SOCIALIS, 3(5), 110-127. http://dx.doi.org/10.35381/racji.v3i5.171

Amar, J., \& Berdugo de Gómez, M. (2011). Vínculos de apego en niños víctimas de la violencia intrafamiliar [Attachment links in child victims of domestic violence ]. Psicología desde el Caribe,, 1-22. Obtenido de https://n9.cl/cah8

Ames, P., \& Crisóstomo, M. (2019). Formas de prevención y atención de la violencia contra las mujeres, niños y niñas en zonas rurales: revisión comparada y estudio de caso en Huancavelica, Perú. Obtenido de https://n9.cl/23jn

Argudo-Tello, K. J., Erazo-Álvarez, J. C., \& Narváez-Zurita, C. I. (2019). Evaluación de Control Interno en Riesgos Estratégicos para la Dirección de Planificación de la Universidad de Cuenca. [Evaluation of Internal Control in Strategic Risks for the Planning Direction of the University of Cuenca]. Revista Arbitrada Interdisciplinaria Koinonía, 67-96. doi:http://dx.doi.org/10.35381/r.k.v4i1.372 
Calle-Abril, D. R., Erazo-Álvarez, J. C., \& Narváez-Zurita, C. I. (2020). Estrategias de mediación y solución de conflictos para el sector industrial de pinturas [Mediation and conflict resolution strategies for the paint industry]. Revista Arbitrada Interdisciplinaria Koinonía, 514-544. doi:http://dx.doi.org/10.35381/r.k.v5i10.704

Casavilca, E. (2019). Violencia familiar y desarrollo del proceso cognitivo en las víctimas de violencia. Obtenido de https://n9.cl/ph6b

Codigo Organico Integral Penal [COIP ]. (2014). Obtenido de https://n9.cl/g6sc

Conger, R. D. (2003). Comportamiento enojado y agresivo en tres generaciones: un estudio prospectivo y longitudinal de padres e hijos. [Angry and aggressive behavior across three generations: A prospective,longitudinal study of parents and children]. Journal of abnormal child psychology., 31(2), 143-160. Obtenido de https://n9.cl/17rw

Erazo, J. C., \& Narváez, C. I. (2020). La gestión del capital intelectual y su impacto en la efectividad organizacional de la industria de cuero y calzado en la Provincia de Tungurahua - Ecuador [The management of intellectual capital and its impact on the ] Recuperado de: https://n9.cl/52li. Revista Espacios, 254-271.

Espinar-Ruiz, E. (2003). Violencia de género y procesos de empobrecimiento: Estudio de la violencia contra las mujeres por parte de su pareja o ex-pareja sentimental. [Gender violence and impoverishment processes: Study of violence against women by their partner or ex-partner ]. Universidad de Alicante. Obtenido de https://n9.cl/35fqz

Guillén, N. P. (2004). Relaciones de poder: leyendo a Foucault desde la perspectiva de género [Power relations: reading Foucault from the gender perspective ]. Revista de Ciencias Sociales (Cr), 4(106), 123-141. Obtenido de https://n9.cl/sdzh

Hernández, O. B. (2018). Desarrollo e implementación de dinámicas para crear habilidades de afrontamiento en adolescentes con violencia intrafamiliar. PsicoEducativa: reflexiones y propuestas, 4(8), 25-33. Obtenido de https://n9.cl/j4fx

López, G., \& Guiamaro, Y. (2017). El rol de la familia en los procesos de educación y desarrollo humano de los niños y niñas [The role of the family in the processes of education and human development of boys and girls ]. Ixaya. Revista Universitaria de Desarrollo Social., 10, 31-55. Obtenido de https://n9.cl/ps42u 
Maldonado-García, V., Erazo-Álvarez, J., Pozo-Cabrera, E., \& Narváez-Zurita, C. (2020). Violencia económica y patrimonial. Acceso a una vida libre de violencia a las mujeres. [Economic and patrimonial violence. Access to a life free of violence against women]. IUSTITIA SOCIALIS, 5(8), 511-526. http://dx.doi.org/10.35381/racji.v5i8.588

McClennen, J. A., \& Day, M. (2016). Social work and family violence: Theories, assessment, and intervention [Trabajo social y violencia familiar: teorías, evaluación e intervención]. New York: Springer Publishing Company. Obtenido de https://n9.cl/wdm1

Mendieta-Ortega, M. P., Erazo-Álvarez, J. C., \& Narváez-Zurita, C. I. (2020). Gestión por competencias: herramienta clave para el rendimiento laboral del talento humano del sector hospitalario [Management by competences: key tool for the labor performance of the human talent of the hospital sector]. Revista Arbitrada Interdisciplinaria Koinonía, 287-312. doi:http://dx.doi.org/10.35381/r.k.v5i10.696

Ministerio de Educacion del Ecuador. (2019). Ministry of Education. Obtenido de https://educacion.gob.ec/

Navarrete, L. P. (2011). Estilos de crianza y calidad de vida en padres de preadolescentes que presentan conductas disruptivas en el aula [Parenting styles and quality of life in parents of preadolescents who present disruptive behaviors in the classroom ]. Obtenido de https://n9.cl/z87i

Rico, M. N. (1996). Violencia de género: un problema de derechos humanos. [Gender violence: a human rights problem ]. México: Cepal. Obtenido de https://n9.cl/1w7s 